\title{
Document-Oriented Heterogeneous Business Process Integration through Collaborative E-Marketplace
}

\author{
Jingzhi Guo, Zhuo Hu, Chi-Kit Chan, Yufeng Luo and Chun Chan \\ Department of Computer and Information Science, University of Macau \\ Av. Padre Tomás, Pereira, S.J., Taipa, Macau \\ Tel: +853-8397 4890
}

\{jzguo, ma66571, ma56518, ma56572, ma46507\}@umac.mo

\begin{abstract}
In this paper, we studied the semantic consistency maintenance issue between heterogeneous contexts, that is, how a firm' business process of one e-marketplace can be transformed to another firm's business process of another e-marketplace in a semantically consistent way. The proposed solution of this paper uses XML Product Map (XPM) of collaborative concept to represent semantically consistent business processes, and adopts common action concept pool and XPM documents to design heterogeneous business processes that are suitable for heterogeneous business process transformation. We motivated the approach with a realworld PVC poncho trade problem and explained it in architecture of collaborative process design and automatic service provision. We reported the implementation specification within a hybrid human-agent framework, where four layers of system modules are specified. The approach is evaluated based on a new semantic impact chain method particularly for evaluating concept consistency in meaning representation between heterogeneous contexts of business processes.
\end{abstract}

\section{Categories and Subject Descriptors}

H.3.4 [Systems and Software]: Information networks; H.3.5 [Online Information Services]: Commercial services, Data sharing, Web-based services; I.2.4 [Knowledge Representation Formalisms and Methods]: Representations; H.4.1 [Office Automation]: Groupware, Workflow management

\section{General Terms}

Design, Languages, Theory

\section{Keywords}

Business process, collaborative process exchange, COPEX, concept, collaborative concept exchange, CONEX, collaborative document exchange, CODEX, electronic marketplace, integration.

\section{INTRODUCTION}

Business process is an important research area in e-commerce. A better understanding of business process helps us create automatic enterprises [3], enable e-business collaboration between firms [14], enhance customer relationship [11], forge stronger supply

Permission to make digital or hard copies of all or part of this work for personal or classroom use is granted without fee provided that copies are not made or distributed for profit or commercial advantage and that copies bear this notice and the full citation on the first page. To copy otherwise, or republish, to post on servers or to redistribute to lists, requires prior specific permission and/or a fee.

10th Int. Conf. on Electronic Commerce (ICEC) '08 Innsbruck, Austria Copyright 2008 ACM 978-1-60558-075-3/08/08 ...\$5.00. chain [15] and improve business knowledge management [2].

In general, business processes are defined as "a set of logically related tasks performed to achieve a defined business task" [4]. Technically, business process is often defined as a collection of activities, where each activity composes an input, a method and an output. By this definition, business process standards are often promoted to enable business activities to be performed between business partners. For example, popular business process standards can be found in OMG.org (e.g. BMM, BPDM and BPMN), OASIS-open.org (e.g. WSBPEL), Microsoft (e.g. BizTalk), and ebXML.org (e.g. ebBP). These business process languages assume that business partners are able to interoperate with each other in their given business process standards.

In real-world business practices, a business process proceeds forward in a way of exchanging different business documents between known or unknown trading partners. For example, a trading process is initiated from sending an inquiry sheet by the inquirer to potential bidders. Then if any bidder is interested in the received inquiry, it will make an offer and send back to the inquirer. After that, counteroffers may follow back and forth between the inquirer and the bidder until the process stops or the acceptance is made by either the inquirer or the bidder for writing the formal contract to legally bind what they have agreed. This trading process implies that the essence of a business process is the exchange of different yet causally related documents such that an antecedent business document is an input of an action that produces a posterior business document. Very obviously, many actions between different antecedent and posterior documents constitute a business process. Another implication of this example is that the used documents between the inquirer and the bidders may be different in formats and term usages. In a traditional paperbased business process, it may not be a problem because a received business document, though it may be different in term usages, human can find out ways to flexibly interpret them exactly or send back for clarification. However, in a modern electronic business process, it will be a problem because computers cannot disambiguate term meanings without any predefined rules.

In the rest of this section, we will exemplify the problem of ebusiness process by describing a real PVC poncho trade process.

\subsection{Document-Oriented Business Process}

A traditional document-oriented business process helps us understand how our e-business processes should be designed and how semantic consistency between heterogeneous e-business processes in different contexts should be maintained.

In Figure 1, we describe a traditional case of importing PVC ponchos from a Chinese exporter to a U.S. importer to illustrate the traditional document-oriented business process. 


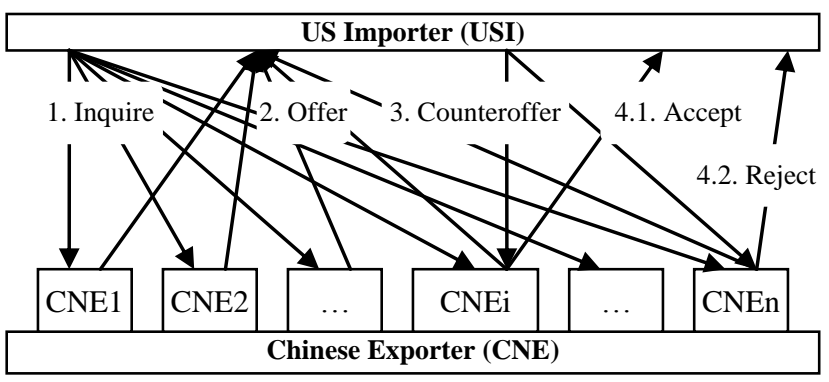

Figure 1: PVC Poncho Trade Process

In this example, the US importer USI sends a Inquire(PVC poncho) to all available Chinese exporters CNE1...CNEn using an inquiry sheet shown in Table 1 .

Table 1: PVC Poncho Inquiry Sheet

\begin{tabular}{|l|l|}
\hline \multicolumn{2}{|c|}{ Inquiry Sheet } \\
\hline Product name & PVC Poncho \\
\hline \multicolumn{2}{|c|}{ Specification } \\
\hline Size & 50 " x 80" \\
\hline Color & Orange \\
\hline Thickness & $0.10 \mathrm{~mm}$ \\
\hline Quantity & 55,000 pieces \\
\hline Shipment & FOB China Main Ports \\
\hline
\end{tabular}

When all rainware-related CNEs receive the inquiry, some of them have PVC poncho products and make the corresponding offers. Among these companies, all have two offering prices, that is, one is the minimum order quantity from 3000 to 5000 pieces and another is the normal export order quantity from 45000 to 60000 pieces. So for CNEs asking minimum export order quantity less than 55000 pieces, they quote prices from USD0.145/piece to USD0.155/piece. For CNEs with minimum export order quantity larger than 55000 pieces, they quote prices from USD0.17/piece to USD0.185/piece. A typical PVC poncho offer sheet from CNEi is shown in Table 2, where some CNE may only make offer in Chinese (note: this is very often in real practice).

Table 2: PVC Poncho Offer Sheet

\begin{tabular}{|c|c|}
\hline \multicolumn{2}{|r|}{ Offer Sheet (报价单) } \\
\hline Product name & PVC Poncho (PVC 斗蓬) \\
\hline \multicolumn{2}{|r|}{ Specification (规格) } \\
\hline Size (尺寸) & $50 ”$ x 80” (127 X 203.2 公分 $)$ \\
\hline Color (颜色) & Orange (桔黄色) \\
\hline Thickness (厚度) & $0.10 \mathrm{~mm}(0.10$ 毫米) \\
\hline Quantity (数量) & 55,000 pieces (55000 件) \\
\hline Shipment (装运条款) & FOB Ningbo, China (FOB 宁波) \\
\hline $\begin{array}{l}\text { Delivery Date } \\
\text { (发运日期) }\end{array}$ & $\begin{array}{l}45 \text { days upon receipt of L/C } \\
\text { (收到信用证后 } 45 \text { 天发货) }\end{array}$ \\
\hline Price (价格条款) & FOB USD0.15/piece (件) Ningbo (宁波) \\
\hline Packing (包装条款) & (details omitted here) \\
\hline Payment (支付条款) & L/C at sight (or 即期信用证) \\
\hline Quality (订货数量) & As per confirmed sample(根据所确认的样品) \\
\hline
\end{tabular}

When the USI receives all the offers, it compares the offers and selects a best offer to further negotiate by sending out counteroffer 1 to the selected CNE, typically shown in Table 3.

Table 3: PVC Poncho Counteroffer 1

\begin{tabular}{|l|l|}
\hline \multicolumn{2}{|c|}{ Offer Sheet } \\
\hline Product name & PVC Poncho \\
\hline \multicolumn{2}{|c|}{ Specification } \\
\hline Size & 50 " x 80" \\
\hline Color & Orange \\
\hline
\end{tabular}

\begin{tabular}{|l|l|}
\hline Thickness & $0.10 \mathrm{~mm}$ \\
\hline Quantity & 55,000 pieces \\
\hline Shipment & FOB Shanghai, China \\
\hline Delivery Date & 30 days upon receipt of L/C \\
\hline Price & FOB USD0.14/piece Shanghai \\
\hline Packing & (details omitted here) \\
\hline Payment & L/C at 45 days \\
\hline Quality & As per the confirmed sample \\
\hline
\end{tabular}

After receiving the counteroffer, the selected CNE may directly accept the counteroffer to conclude the deal or further negotiate with USI by sending back the counteroffer 2 shown in Table 4 .

Table 4: PVC Poncho Counteroffer 2

\begin{tabular}{|l|l|}
\hline \multicolumn{2}{|c|}{ Offer Sheet (note: the possibility in Chinese is omitted) } \\
\hline Product name & PVC Poncho \\
\hline \multicolumn{2}{|c|}{ Specification } \\
\hline Size & 50 " x 80 " \\
\hline Color & Orange \\
\hline Thickness & $0.10 \mathrm{~mm}$ \\
\hline Quantity & 55,000 pieces \\
\hline Shipment & FOB Shanghai, China \\
\hline Delivery Date & 30 days upon receipt of L/C \\
\hline Price & FOB USD0.142/piece Shanghai \\
\hline Packing & (details omitted here) \\
\hline Payment & L/C at sight \\
\hline Quality & As per the confirmed sample \\
\hline
\end{tabular}

If there is no direct acceptance from the counteroffer 1, the USI may accept the counteroffer 2 to conclude the deal by sending back a signed purchase order (or simply a confirmation notice with regard to the counteroffer 2), or it may abandon the selected $\mathrm{CNE}$ and go back to the second best offer to restart a negotiation until the deal is done.

Table 1 to Table 4 has described a document-oriented trading process, which is very useful for us to understand the essence of business processes for e-marketplaces. In general, a documentoriented business process is a sequence of conditional actions. Each action is an action concept, which has an action sender and an action receiver. The action acts based on an action logic particular to this action. This action logic acts on a set of objects (here are documents), where some objects provide the input for the action logic and some objects receive the output of the action logic. These objects are consumed by both action sender and action receiver. Generic actions can be diagrammed in Figure 2.

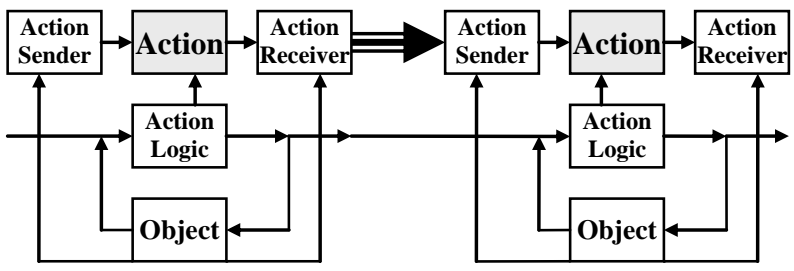

Figure 2: Generic Actions in a Business Process

For example, an action sender USI sends a document of inquiry sheet to action receivers CNEs through an action of Inquiry(PVC poncho). When the action receivers $\mathrm{CNEs}$ receive the inquiry sheet, they turn themselves into action senders and make the action of Offer(PVC poncho) on another document of offer sheet.

If a traditional document-oriented business process (TDOBP) has been converted to an electronic document-oriented business process (EDOBP), we will at once have serious issues when we send an action concept from USI to CNEs or vice versa as per Figure 2. 


\subsection{Technical Issues in EDOBP}

The issues of converting TDOBP to EDOBP are:

First, unlike human who can intuitively read and understand the exchanged letters and facsimiles even if they are situated in heterogeneous contexts, computers must have definite and shared concepts to correctly receive and interpret the exchanged electronic documents. For example, computer A and B cannot understand that "PVC poncho" is a product if it is not defined as a product name.

Second, human can easily understand the different semantic terms in documents that are created in different contexts, but computers cannot resolve them if no mapping rules are provided. For example, if the inquirer's computer uses "Polyvinyl Chloride poncho" and the bidder's computer only understands the term "PVC poncho", the trading process then cannot proceed from Inquire action to Offer action.

Third, human can easily read and act on documents using their intuitions (i.e. human action logics), but computers cannot read and act on documents if no given action logics are provided. For example, when human receives an inquiry letter, s/he can quickly react how to process it. For a computer, if there are no given rules (e.g. Inquire follows by Offer), it will not automatically process inquiry sheet to make offer.

To resolve the above semantic consistency issues for enabling EDOBP that can be used for constructing e-marketplaces that have characteristics of distribution, autonomy, emergence and interdependence [10], these phenomenal issues should be further abstracted into two technical integration issues as follows:

- Heterogeneous business processes of different contexts must have a semantically consistent common vocabulary.

- Heterogeneous business processes of different contexts must understand the actions with each other when they interact, so that they can freely match interacting behaviors to enable a cross-context business process.

This paper aims to solve the above two technical issues by proposing a novel approach called collaborative process exchange (COPEX). This approach regards a business process as a conditional sequence of automated actions on a set of business documents, where business concepts are collaboratively created and maintained for resolving semantic conflicts.

Nevertheless, semantic consistency maintenance between heterogeneous business processes is a rather new research issue in the area of e-Commerce. Currently, few works could be found except in the authors' research groups. Thus, the theme of this paper mainly relates to CONEX [7] and CODEX [8], which are early works of the authors. They focus on how to represent heterogeneous concepts and how to apply collaboration method to maintain semantic consistency between heterogeneous contexts.

Business process standards are a way of achieving business process interoperation and collaboration, for example, WSBPEL, ebBP and BizTalk. However, these standards themselves do not resolve semantic conconsistency issues. Thus, standardization approach is different from the proposed COPEX appraoch, which promotes collaboration as a key way of maintaining semantic consistency between heterogeneous business processes.

In addition, evaluation method on semantic consistency maintenance lacks in current research, though there are some remotely related works of ontology evaluation methods (e.g. Leukel et al's research group [12]) in ontology design field. But it seems that their task is different from the evaluation of semantic consistency and mainly focuses on evaluating the quality of ontology structures that are in use for standardization. Thus, another aim of this paper is to provide a new evaluation method on semantic consistency maintenance.

The rest of the paper is arranged as follows: Section 2 provides an overview the technology used in this paper. Section 3 describes the collaborative concept exchange approach. Section 4 provides the implementation specification by the example of PVC poncho trade. In Section 5, a new evaluation method particularly for semantic consistency maintenance is given. Finally, a summary of the paper, the advantages the proposed approach, and the future works are given in conclusion.

\section{OVERVIEW OF USED TECHNOLOGY}

The collaborative process exchange (COPEX) approach applies the technology of collaborative conceptualization.

Collaborative conceptualization is a collaboration-based and concept-centric business information representation and design approach to maintaining semantic consistencies between heterogeneous business information by collaborative concepts [7][8][9]. It can represent and integrate heterogeneous business vocabularies [7] and business documents [8]. It was developed from the ideas of semiotics (e.g. [1][13][16]), context analysis (e.g. [5]) and collaborative editing (e.g. [17]). The main idea is that information in heterogeneous structures, semantics and contexts can be kept semantically consistent if they can be represented in PRODUCT MAP and collaboratively created and maintained [9]. The key benefit of this approach is its elimination of semantic conflicts amongst heterogeneous business information contexts and enabling crossdomain interactions.

The main features of this approach are: (1) Structure, concept and context of information representation are independently separated without interweaving each other, (2) a semantic consistency maintenance model guarantees that heterogeneous structures are mappable, heterogeneous concepts are semantically equivalent and heterogeneous contexts are transformable, and (3) collaboration mechanisms ensure that the semantic consistency model is implementable.

The basics of collaborative conceptualization can be expressed as follows along with a PRODUCT MAP theory [7]:

- A PRODUCT MAP PM is a representation and is defined as a set of recursive $\mathrm{PM}=\left(\mathrm{PM}_{1}, \ldots, \mathrm{PM}_{\mathrm{n}}\right)$ in which $\mathrm{PM}_{\mathrm{i}}=(S, C, X) \rightarrow I$, where $S$ is structure, $C$ is concept, $X$ is context and $I$ is reified concept. $S$ can be represented in anyway, e.g. (IID = "unique identifier"; $\mathrm{AN}=$ "annotation"; $\mathrm{LK}=$ "link to context"; OP = "a set of optional elementary structures"), $C$ can be any natural language annotation for defining the concept, and $X$ can refer to any contextual semantic community, e.g. in the form of domain namespace.

- Structure is meaningless if no concept is conveyed. For example, in a given context $x=$ "boo.com", a structure $S i=(i i d$, an, $x=$ "boo.com") means nothing but a structural symbol.

- A meaningless structure $S_{i}$ can be conceptualized as a concept $C_{i}$ by conveying a meaning. For example, the structure $S_{i}$ is meaningful only if it is conveyed with a meaning e.g. $C_{i}=$ "color" uniquely identified as iid="1501.3" such that $\left(C_{i} \Rightarrow S_{i}\right) \Leftrightarrow$ (iid="1501.3", an="color", $x=$ "boo.com"). Here $C_{i}$ causally determines how $S_{i}$ is conveyed with a concept. 
- A concept $C_{i}$ can be instantiated into a reified concept $I_{i}$. E.g., a concept $C_{i}=$ (iid = "1501.3", an = "color", $x=$ "boo.com") can be instantiated into a reified concept $C_{i} \rightarrow$ ( $I_{i}=$ "red").

- Given a collaboration mechanism $\Gamma$, any two $\mathrm{PM}_{1}$ and $\mathrm{PM}_{2}$ in two heterogeneous contexts $X_{1}$ and $X_{2}$ are semantically consistent if and only if they are (1) structurally mappable such that $\operatorname{map}\left(S_{1} @ X_{1}, S_{2} @ X_{2}\right)$ on $\Gamma$, (2) semantically equivalent such that $\left(C_{1} @ X_{1}={ }_{\text {sem }} C_{2} @ X_{2}\right)$ on $\Gamma$, and (3) contextually common through a common context $\Gamma$ such that $\left(X_{1} @ \Gamma \in \Gamma\right) \cap\left(X_{2} @ \Gamma \in \Gamma\right)$ where collaboration mechanism $\Gamma$ facilitates as a common context.

The formal PRoduct MAP is defined as the Grammar of CONEX Structure Model in [7]. The collaboration mechanism $\Gamma$ is devised as several collaborative engines of CONEX in [7][9].

\section{COPEX APPROACH}

COllaborative Process EXchange (COPEX) approach has three design principles [7]: (1) Flexibility: the efficacy of COPEX system shall not degrade drastically when the number of participated heterogeneous business processes increases and when personalization of individual business processes is required. (2) Exactness: COPEX system shall be able to maintain semantic consistency of business concepts between heterogeneous business processes that are participated. (3) Evolvability: COPEX system shall be able to insert new business concepts and modify the existing business concepts without the global effects to the existing participated systems in use.

With these principles, this paper designs the COPEX approach in three parts: proposing the collaborative business process design architecture, providing a common action concept pool for flexible business process personalization, and devising an electronic document-oriented business process for maintaining semantic consistency of heterogeneous business process patterns.

\subsection{CPDASP Model}

Collaborative process design and automatic service provision (CPDASP) is a way of collaboratively designing and using semantically consistent business processes between business process providers and e-marketplace participants so that business process users can obtain desired transaction results. For example, the USI business system can interact with the CNE business systems for inquiring and offering PVC ponchos in a semantically consistent way. The CPDASP involves several steps as follows:

(1) E-marketplace facilitators (EMF) collaboratively design document-oriented business processes in the way of collaborative design of (a) common business vocabularies $\mathrm{V}(\mathrm{X})$, (b) common business document templates $\mathrm{D}(\mathrm{X})$ by using $\mathrm{V}(\mathrm{X})$, and (c) common business process patterns $\mathrm{P}(\mathrm{X})$ by using $\mathrm{V}(\mathrm{X})$ and $\mathrm{D}(\mathrm{X})$.

(2) Firms (e.g. USI and CNEs) (FIRM) localize V(X), D(X) and $\mathrm{P}(\mathrm{X})$ into their own personalized firm-based $\mathrm{V}^{\prime}(\mathrm{X}), \mathrm{D}^{\prime}(\mathrm{X})$ and $\mathrm{P}^{\prime}(\mathrm{X})$ to satisfy their own firm preferences and requirements through a collaborative mapping such that:

- $\forall \mathrm{t}^{\prime} \in \mathrm{V}^{\prime}(\mathrm{X}), \forall \mathrm{t} \in \mathrm{V}(\mathrm{Y}) \subseteq \mathrm{V}(\mathrm{X}) \bullet \mathrm{t}^{\prime}{ }{ }_{\mathrm{sem}} \mathrm{t}$;

- $\forall \mathrm{d}^{\prime} \in \mathrm{D}^{\prime}(\mathrm{X}), \forall \mathrm{d} \in \mathrm{D}(\mathrm{Y}) \subseteq \mathrm{D}(\mathrm{X}) \bullet \mathrm{d}^{\prime}{ }_{\text {sem }} \mathrm{d}$ if and only if $\forall$ td' $\in$ d', td' $\in \mathrm{t}^{\prime}$ and $\forall \mathrm{td} \in \mathrm{d}$, td $\in \mathrm{t}$;

- $\quad \forall \mathrm{p}^{\prime} \in \mathrm{P}^{\prime}(\mathrm{X}), \forall \mathrm{p} \in \mathrm{P}(\mathrm{Y}) \subseteq \mathrm{P}(\mathrm{X}) \bullet \mathrm{p}^{\prime}={ }_{\text {sem }} \mathrm{p}$ if and only if $\forall$ tp' $\in$ p', tp' $\in$ t' and $\forall$ tp $\in$ p, tp $\in$ t.

The notation " $=$ sem" means semantically equivalent, for example, "refrigerator" "= $=$ sem "fridge", and "Quote('refrigerators')" = "Offer('fridge')" after collaborative agreement.
(3) Users of FIRM subscribe and use EMF-provided services to automatically transform heterogeneous business processes from one firm to another firm.

The CPDASP Model is diagrammed in Figure 3, which is on a distributed P2P/D2F collaboration network discussed in [9]. P2P refers to the peer-to-peer collaboration network, where many EMF collaboratively design and maintain common business concepts of common business vocabularies (comVoc), common document template (comDoc) and common business processes patterns (comProc). For each common concept created in different EMF, it has the same and unique concept identifier but its concept annotation may be different yet with the same consistent meaning in different EMF. D2F means dominant-to-follower ${ }^{l}$ collaboration network, which is point-to-point. In this D2F architecture, different FIRM localize comVoc, comDoc and comProc of EMF into their personalized firm-wide business vocabularies (firmVoc), firm-wide business document templates (firmDoc) and firm-wide business process patterns (firmProc). A firm-wide concept may have a different concept identifier from common concept but it is mapped onto the common concept identifier to refer to a same semantically consistent concept.

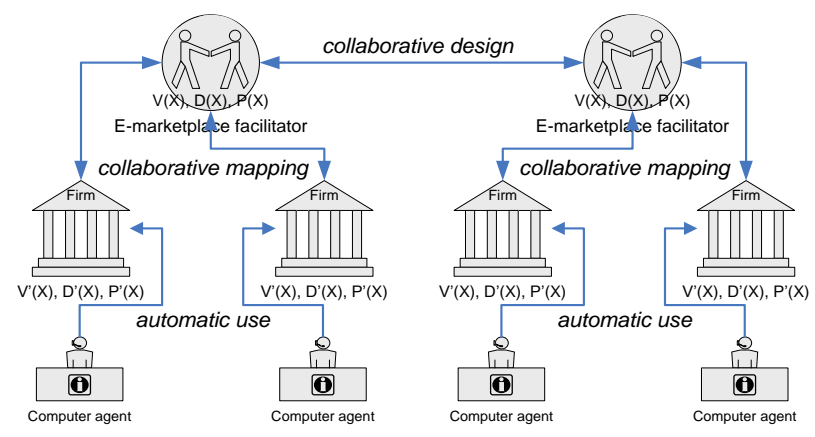

Figure 3: CPDASP Model

\subsection{Common Action Concept Pool}

With CPDASP Model, this subsection describes a particular novel method to maintain semantic consistency between heterogeneous business processes of different contexts so that business process in one firm of one e-marketplace can be transformed to another firm of another e-marketplace.

\subsubsection{Business Concept Classification}

A business process consists of many complex concepts. In order to understand what are the essential components of a generic business process, business concepts are classified into categories of basic concepts, composite concepts, action concepts and reified concepts.

- Basic concepts (BC), which are noun-form class concepts in terms of a category, a class or a physical or abstract phenomenon. For example, product terms are basic concepts.

- Composite concepts (CC), which are noun-form concepts but they are composed by a set of logically related basic concepts. Any document templates are composite concepts.

- Action concepts (AC), which are verb-form concepts that compose actions. For each action concept, the action has its

\footnotetext{
${ }^{1}$ Note: dominant refers to knowledge engineers qualified as experts to create global business knowledge, while follower refers to knowledge engineers not qualified as experts to create global knowledge
} 
fixedly designated action sender, receiver, logic, and targeted objects.

- Reified concepts (RC), which are concept instances that associate concepts of $\mathrm{BC}, \mathrm{CC}$ and $\mathrm{AC}$ with symbols. A symbol is an independent representation, which is meaningless by its own, e.g. "33", "red", computing formulas or picture. Symbols are meaningful only if they are associated with concepts of $\mathrm{BC}, \mathrm{CC}$ and $\mathrm{AC}$. However, in reality, symbols often have their fixed social meanings with regard to our real-world interpretation. Particular in this paper, we say symbols are meaningless only because they are not collaboratively designed in CPDASP model and their meanings are not clarified for consistent use. BC, CC and AC can all have their $\mathrm{RC}$ when users instantiate them.

The classification of business concepts allows us to separate the designs and use of business concepts in CPDASP model. In concept design stage, collaborative basic concepts are used to design semantically consistent business vocabularies. Composite concepts are collaboratively designed as semantically consistent business document templates using the designed basic concepts. Action concepts are collaboratively designed as semantically consistent components of business process patterns using both basic and composite concepts. In concept use stage, reified concepts of basic, composite and action concepts are created and used by FIRM users through combining concepts and symbols.

\subsubsection{Action Concept Pool}

We design a novel business process as a conditional sequence of action concepts, that is, a business process can be freely reassembled using different action concepts from an action concept pool based on the desired conditions for triggering each action concept that becomes the part of the business process.

For example, given an action concept pool:

\{

LaunchInquiryProcess(), MakeInquiry(t, d), ApproveInquiry(t, d), ApprovedInquiry(t,d), RejectedInquiry(t, d), ReadInquiry(t, d), EditInquiry(t, d), SendInquiry(t, d), ReceiveInquiry(t, d), MatchInquiry(t, d), ReadMatchedInquiry(t, d), EditMatchInquiry(t, d), StopInquiry(t, d), AnswerInquiry(t, d), LaunchOfferProcess(), MakeOffer(t, d), ApprovedOffer(t, d), RejectedOffer(t, d), ReadOffer(t, d), EditOffer(t, d), StopOffer(t, d), SendOffer(t, d), ReceiveOffer(t, d), SendInquiryAnswer(t, d), ReceiveInquiryAnswer( $\mathrm{t}, \mathrm{d})$ \} ,

where both inquirer and bidder can freely assemble their Inquire and Offer business processes as they wish.

With the above action concept pool, the US importer USI can configure an Inquire process as follows if there is no internal approval needed for any product inquiry:

LaunchInquiryProcess ()$\rightarrow$ MakeInquiry(PVC poncho, USI inquiry document template) $\rightarrow$ EditInquiry(PVC poncho, USI inquiry document instance) $\rightarrow$ SendInquiry(PVC poncho, USI inquiry document instance).

For Chinese exporters CNEs, CNE1 may use automatic inquiry match to make offer as follows:

ReceiveInquiry(PVC poncho, CNE1 inquiry document instance) $\rightarrow$ MatchInquiry(PVC poncho, CNE1 match document template $\rightarrow$ MakeOffer(PVC poncho, CNE1 offer document template) if Inquiry is satisfied, or AnswerInquiry(PVC poncho, failed inquiry answer document template) if Inquiry is not satisfied $\rightarrow$ SendOffer(PVC poncho, CNE1 offer document instance) if satisfied, or SendInquiryAnswer(PVC poncho, CNE1 failed inquiry document instance).

Similarly, other Chinese exporters CNEi can configure their own Offer processes in different ways using the action concept pool.

Obviously, the action concept pool has provided a highly flexible and personalized way of configuring business processes in different firms that may have heterogeneous process patterns. However, to realize the action concept pool, two detailed technical issues need to be resolved.

- Different firms (e.g. USI and CNEi) must share a common action concept pool so their devised patterns of business processes have semantic commonality for interoperability.

- Different firms (e.g. USI and CNEi) have their heterogeneous business contexts. It means that they may have different business vocabularies, business document templates and business process patterns.

In this paper, the CPDASP architecture discussed in Section 3.1 solves the above two problems. First, the common business vocabularies (comVoc), common business document templates (comDoc) and common business process patterns (comProc) collaboratively designed between EMF ensure that firms (e.g. USI and $\mathrm{CNEi}$ ) located in different e-marketplaces could share sets of semantically consistent basic concepts, composite concepts and action concepts. It assures that a shared common action concept pool is available to all participated firms. Second, the D2F collaboration mechanism for collaborative mapping local concepts of firms onto common concepts ensure that firm-wide business vocabularies (firmVoc), business document templates (firmDoc) and business process patterns (firmProc) in heterogeneous contexts are transformable and understandable with each other.

\subsection{E-Document Oriented Business Process}

Electronic document oriented business process (EDOBP) describes how heterogeneous business process patterns can be integrated for transforming from one business context (e.g. USI) to another (e.g. CNEi) in a semantically consistent way using the common action concept pool to exchange action concepts acted on PRODUCT MAP documents within the architecture of collaborative concept design.

\subsubsection{PRODUCT MAP Based Process Representation}

To maintain semantic consistency between heterogeneous process patterns, the heterogeneous concepts that compose these business processes must have a way to be semantically transformed. In this paper, PRODUCT MAP, introduced in Section 2, is adopted as the business process representation to realize semantically-consistent business process transformation between heterogeneous contexts.

A PRODUCT MAP based business process representation can be described in Figure 4, which generically represents electronic business processes in PRODUCT MAP representation style. In this representation, a process has its unique identifier "iid" and meaning definition "an" and may refer to (refTo) a business process designed in elsewhere. For all business processes, they belong to a specially designed process vocabulary such that $\forall$ process, process $\in$ PROC. Each process consists of a set of actions such that $\forall$ ac$\operatorname{tion}^{\mathrm{i}} \in$ process $(i \in 1 \ldots n)$, action ${ }^{\mathrm{i}} \in$ PROC. Each action has "iid", "an", "refTo", "vis" for visibility to distinguish between public, 
community and private actions, "sta" for status to distinguish between action concept and action instance, "snd" for action sender, and "rcv" for action receiver. An action acts on a set of business documents "docs" such that $\forall$ doc $\in$ docs. Each "doc" is registered in a document vocabulary DOC and has "iid", "an", "refTo", "vis", "sta" for status to distinguish between document template and document instance, and "attach" to state whether the document is attached or placed elsewhere. All "docs" are processed by "logic" using the "prog" program designed in "url". The "logic" has "iid", "an" and "handle" to tell whether "prog" will automatically or manually run to process the "docs". If a "logic" result is a predefined " $\mathrm{x}_{\mathrm{i}}$ " value, it will automatically trigger a corresponding next action including its document sets filled by the last action.

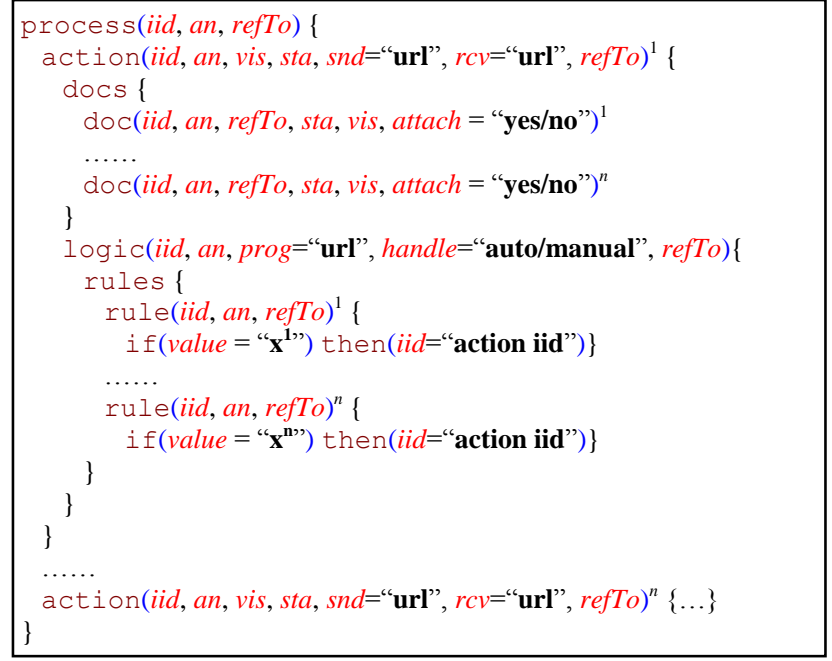

Figure 4: Product Map Based Process Representation

\subsubsection{Business Process Transformation}

Given the PRODUCT MAP based business process representation, each firm in heterogeneous contexts can localize their business processes in a semantically consistent way from the common action concept pool. After the localization, these heterogeneous business processes can be transformed between firms of emarketplaces for business interaction as follows.

(1) Any local action concept "locAction" is mediated by a common action concept "comAction" between SendAction and ReceiveAction such that $\operatorname{map}\left(\mathrm{IID}_{\text {locAction }}\right.$, $\left.\mathrm{IID}_{\text {comAction }}\right)$, translate(vis, sta), remove (an, refTo) and copy(snd, rcv). Here, map() is a swap of IID, translate() is a kind of language translation between different natural languages or dialects used by sender and receiver, remove () is to trim the irrelevant contents for receivers, and copy() is to move contents to the transformed documents.

(2) Any local document "locDoc" is mediated by common document "comDoc" between SendAction and ReceiveAction such that $\operatorname{map}\left(\mathrm{IID}_{\text {locDoc}}, \mathrm{IID}_{\text {comDoc }}\right)$, translate(vis, sta, attach) and remove (an, refTo).

(3) The local logic "locLogic" is mediated by common logic "comLogic" between SendAction and ReceiveAction such that map $\left(\mathrm{IID}_{\text {locLogic }}, \mathrm{IID}_{\text {comLogic }}\right)$, remove(an) and substitute(prog, han- dle, rules) using the locally defined "locLogic". It is worth pointing out that substitute() here uses local action logics to process the incoming actions. This is important because how to process incoming business documents is a local matter and not global. It is able to substitute them because the incoming documents are already understandable after transformation on PRODUCT MAP representation format.

(4) The instances of concepts in constant concept values (e.g. instance "red" of concept "color") are automatically translated between heterogeneous business processes, applying a context-based constant concept value translation approach described in [6]. For example, given a "color" concept with an instance of "orange", it will automatically translate “orange” into “橙色” but not “柑桔” if the receiver is Chinese.

(5) The instances of concepts in computational group concepts (e.g. instance "20 inches" of concept "width") are automatically converted between heterogeneous business processes, applying automatic heterogeneous concept transformation approach described in [7]. For example, "20 inches" in "width" will be automatically converted into “50.8 厘米” in “width".

It is interesting to see that, in fact, for all actions in a given business process, only "public" or "community" actions should be transformed via common level mediation. The "private" actions just stay within the firms. Thus, only outgoing SendAction and incoming ReceiveAction should consider the heterogeneous action transformation. For example, for the whole trading process illustrated in Table 1-4, only SendInquiry(PVC poncho), ReceiveInquiry(PVC poncho), SendAnswerInquiry(PVC poncho), ReceiveAnswerInquiry(PVC poncho), SendOffer(PVC poncho), ReceiveOffer(PVC poncho), SendAcceptance(PVC poncho) and ReceiveAcceptance(PVC poncho) need to be considered. The other actions are internal private actions for both USI and CNEi.

In summary, COPEX approach maintains semantic consistency between any heterogeneous business processes of firms within the collaborative e-marketplaces for firms to correctly interact with each other.

\section{EXAMPLE OF PVC PONCHO TRADE}

We provide a concrete example of PVC poncho trade to see how heterogeneous business processes can be transformed between a US importer USI and a Chinese exporter CNE. In this example, the actions of PVC poncho trade process are represented in XPM (an XML implementation of PRODUCT MAP). Its detailed specification of XPM can be found in [7].

Due to space limitation, we only show the partial process transformation of Inquire actions instead of the whole trade process from Inquire to Accept actions. To simplify the example, we only discuss two public Inquire actions between the USI and the CNE, that is, SendInquiry(PVC poncho) in the USI and ReceiveInquiry( $\mathrm{PVC}$ poncho) in the CNE.

In Figure 5, 6, 7 and 8, we show the heterogeneous Inquire action transformation process from USI to US EMF to China EMF to CNE, such that Figure $5 \rightarrow$ Figure $6 \rightarrow$ Figure $7 \rightarrow$ Figure 8 in a semantically consistent way.

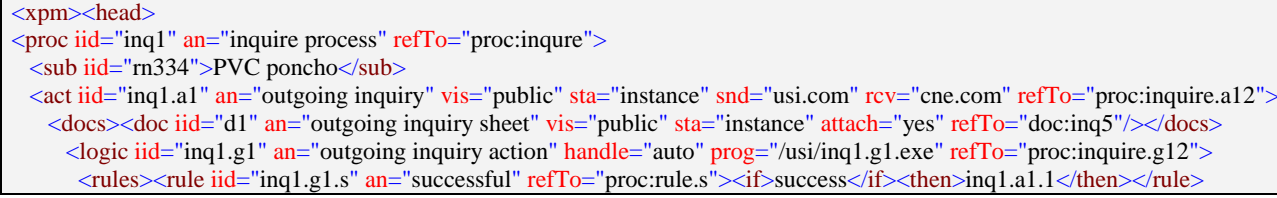




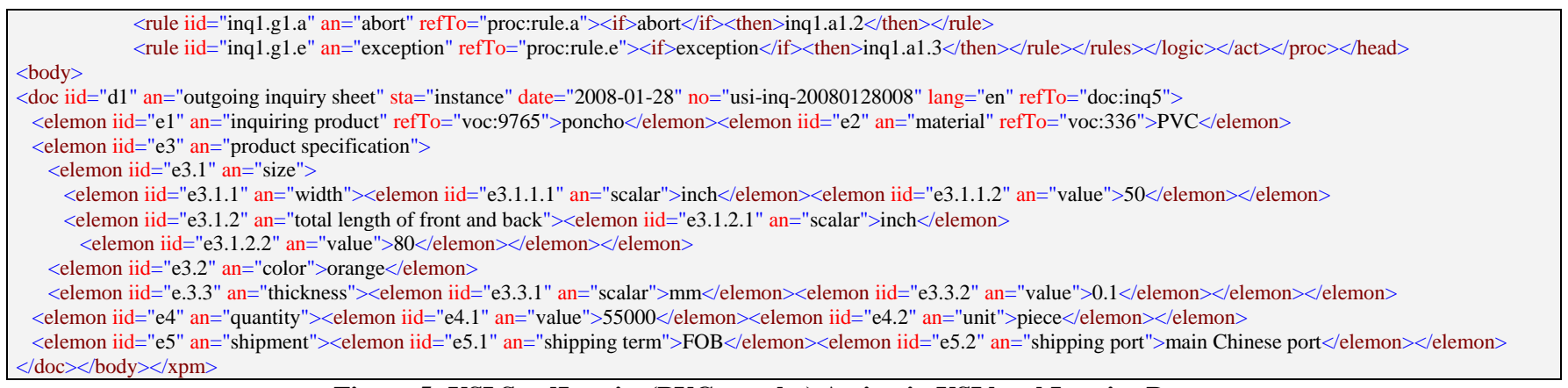

Figure 5: USI SendInquiry(PVC poncho) Action in USI local Inquire Process

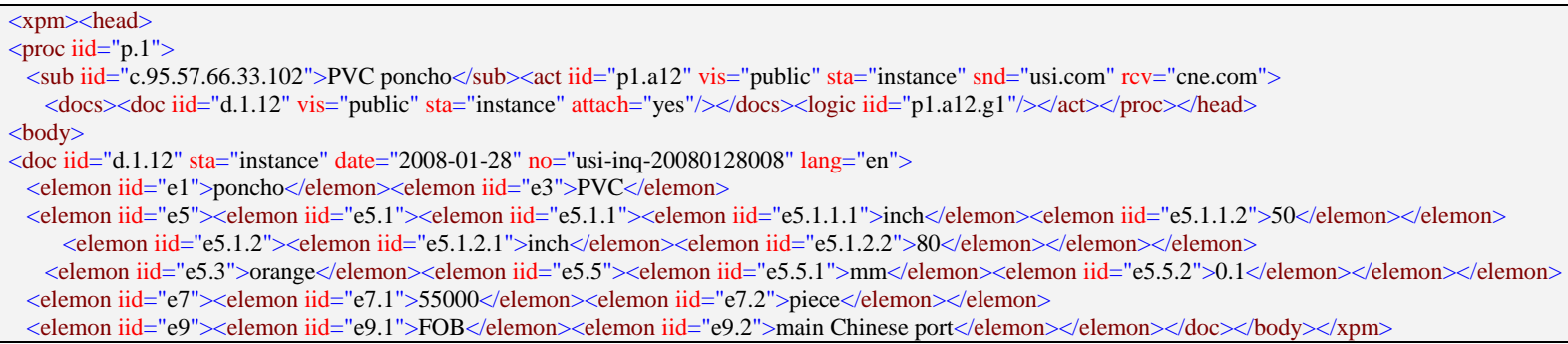

Figure 6: USI SendInquiry(PVC poncho) Action in US EMF Common Inquire Process

$<\mathrm{xpm}>\langle$ head $>$

$\langle$ proc iid="p. $1 ">$

$\langle$ sub iid="c.95.57.66.33.102" >PVC雨披 $</$ sub $>\langle$ act iid="p1.a12" vis="公共" sta="实例" snd="usi.com" rcv="cne.com" $>$

$\langle$ docs $><$ doc iid="d.1.12" vis="公共" sta="实例" attach="yes"/ $></$ docs $><\operatorname{logic}$ iid="p1.a12.g1"/ $></$ act $\rangle</$ proc $></$ head $>$

$\langle$ body>

$<$ doc iid="d.1.12" sta="实例" date="2008-01-28" no="usi-inq-20080128008" lang="cn">

<elemon iid="e1" >雨披</elemon ><elemon iid="e3" >PVC</elemon>

<elemon iid="e5" $>\langle$ elemon iid="e5.1" $><$ elemon iid="e5.1.1" $>\langle$ elemon iid="e5.1.1.1" $>$ 英寸 $</$ elemon $>\langle$ elemon iid="e5.1.1.2" $>50</$ elemon $>\langle/$ elemon $>$

$<$ elemon iid="e5.1.2" ><elemon iid="e5.1.2.1" >英寸 </elemon $><$ elemon iid="e5.1.2.2" $>80</$ elemon $></$ elemon $></$ elemon $>$

<elemon iid="e5.3" > 橙色 $</$ elemon $><$ elemon iid="e5.5" $><$ elemon iid="e5.5.1" $>$ 毫米 $</$ elemon $><$ elemon iid="e5.5.2" $>0.1</$ elemon $>\langle/$ elemon $></$ elemon $>$

$<$ elemon iid="e7" $><$ elemon iid="e7.1" $>55000</$ elemon $><$ elemon iid="e7.2" $>$ 件 $</$ elemon $></$ elemon $>$

<elemon iid="e9" ><elemon iid="e9.1" $>$ FOB $\langle/$ elemon $>\langle$ elemon iid="e9.2" $>$ 中国主要港口 $</$ elemon $\rangle\langle$ elemon $\rangle\langle/$ doc $\rangle\langle/$ body $\rangle\langle/ x p m>$

Figure 7: USI SendInquiry(PVC poncho) Action in Chinese EMF Common Inquire Process

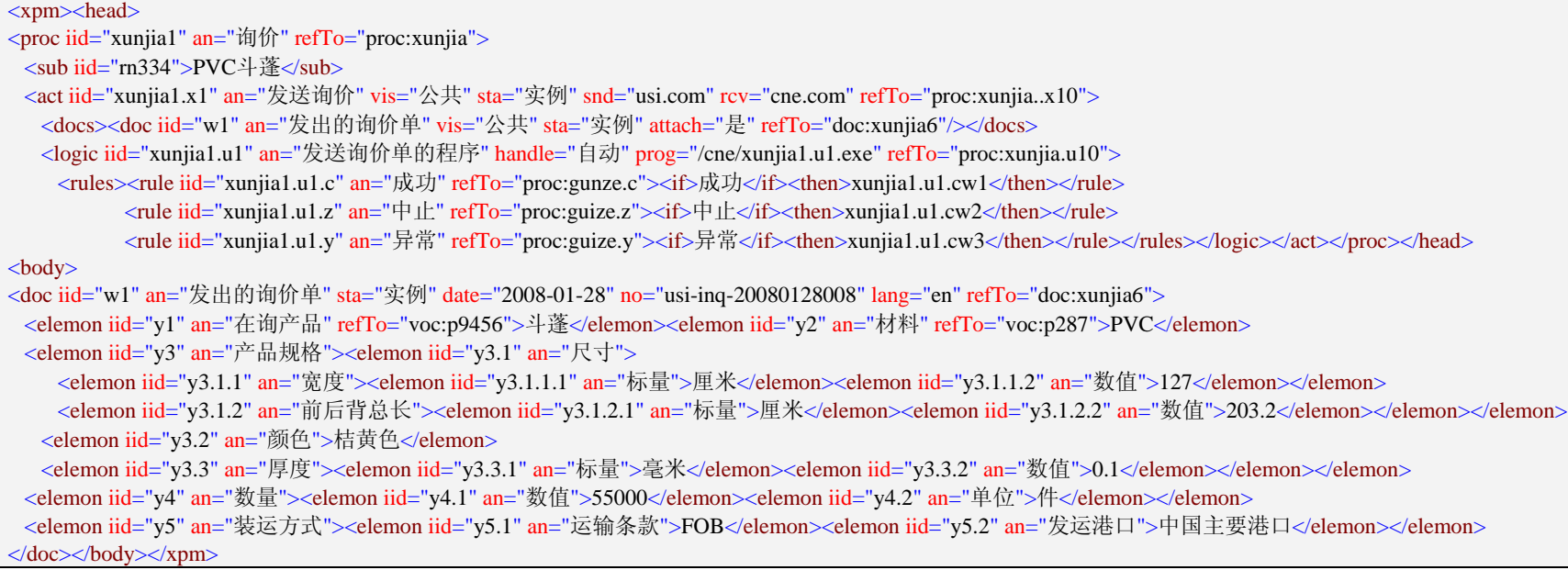

\section{Figure 8: USI SendInquiry(PVC poncho) Action in CNE Local Inquire Process}

Figure 5, 6, 7 and 8 illustrate the four different forms of a SendInquiry(PVC poncho) action <act> of the inquiry process 〈proc>, that is, a part of e-document document oriented trade process made from the common action pool in CPDASP architecture, which are transformed from the local action of USI firm in USA (i.e. Figure 5) into the common action of US EMF (i.e. Figure 6), further into the common action of China EMF (i.e. Figure 7), and finally into the local action of CNE firm in China (i.e. Figure 8).
In this transformation procedure, the actions deliver the product inquiring message using the attached business documents $\langle\mathrm{doc}\rangle$. How to collaboratively design and use business documents can be found in [8].

In the rest of this section, we provide an implementation specification of COPEX system to describe how to implement the above example, following the design principles of flexibility, exactness and evolvability provided in Section 3. 


\subsection{Collaborative Human-Agent Framework} The PVC poncho trade process example involves two types of subsystems of COllaborative Process Editing (COPE) and Automatic Process Transformation (APT) around two categories of business organizations of local firms (FIRM) and $e$ marketplace facilitators (EMF). It requires not only collaboratively designing and maintaining the semantic consistent business concepts in the forms of business vocabularies, business documents and business processes, but also providing automatic heterogeneous business process transformation services. Thus, the whole COPEX systems should be mixed with human and automated agents. To enforce it, this paper adopts a hybrid collaborative human-agent framework, shown in Figure 9.

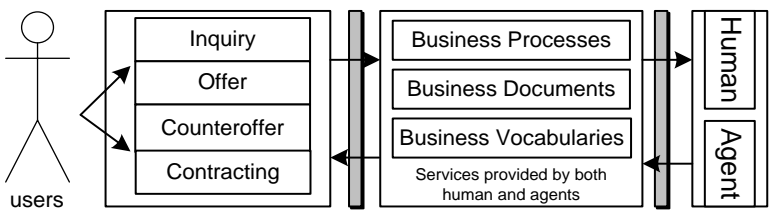

Figure 9: Hybrid Collaborative Human-Agent Framework

In this framework, human designers of both EMF and FIRM are responsible for human-related work, e.g. collaborative designing, editing, modifying, approving and publishing business concepts. Automated agents of both EMF and FIRM are responsible for non-human work, e.g. automatically mapping, matching and forwarding human-provided action concepts. Users of FIRM, on the other hand, simply subscribe and use the services of heterogeneous process transformation that both human designers and automated agents provide.

This framework has benefits of modular design, capability of evolving business concepts and flexibility of increasing the number of both FIRM and EMF. An extra benefit is the semantic consistency maintenance such that the roles of agents have no rights to make erroneous inferences without mutually-agreed collaborative concepts. It prevents semantic conflicts between users and designers and ensures the fulfillment of COPEX goal.

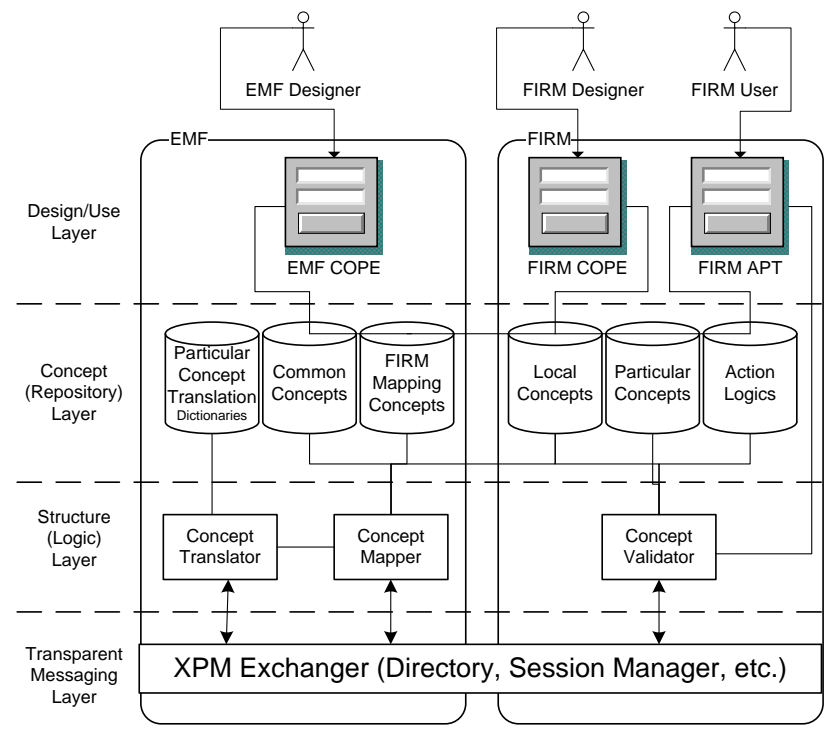

Figure 10: COPEX Architecture

\subsection{System Architecture and Modules}

The COPEX systems work on a distributed P2P/D2F collaboration network, described in Section 3.1. Applying this architecture, the modules of COPEX systems are placed in four layers of design and use, structure logic and transparent messaging, shown in Figure 10.

- The messaging layer is the bottom layer and is responsible for transforming heterogeneous business processes to exchange business concepts between FIRM via EMF in XPM on HTTP protocol. It consists of XPM Exchanger Module in all locations. It consists of components such as search directory of users, FIRM and EMF, and Session Manager for managing interactions.

- The structure (logic) layer is responsible for mapping and translating heterogeneous business concepts from one business organization to another through modules of Concept Translator and Concept Mapper both appeared in EMF. The Concept Validator Module of FIRM parses and validates the XPM representations for transformation.

- The concept (repository) layer is responsible for storing and retrieving various business concepts in both EMF and FIRM. In this layer, Common Concepts are repository collaboratively edited by EMF concept designers using EMF COPE subsystem. Reified Concept Translation Dictionaries are repository edited by an external CAT system (please refer to [6] for CAT systems). Local Concepts, FIRM Mapping Concepts and Action Logics are repositories collaboratively edited by FIRM concept designers through localization mechansim using FIRM COPE subsystem. Reified Concepts are various concept instances created and used by FIRM users with FIRM APT subsystem.

- Design and use layer is the highest layer, which is responsible for the collaborative creation and edition of the common concepts by EMF Designers and the local concepts and action logics by FIRM Designers. In this layer, FIRM users creates reified concepts and use action logics to exchange business information with remote users.

Different roles interact with each other using their own user interfaces provided by the design/use layer. Particularly, EMF designers use EMF COPE editing system as collaborative concept editor, FIRM designers use FIRM COPE editing system as concept localization editor, and FIRM users use FIRM APT transformation system as concept transformer.

\section{EVALUATION OF COPEX SYSTEMS}

In Section 4, flexibility, exactness and evolvability are given as the requirements of system implementation. In the related works of [7], these are also the key requirements for exchanging ad hoc product concepts. In agreement with these requirements, this paper takes the exactness, that is, semantic consistency between heterogeneous business processes as the most important evaluation criterion of COPEX systems. As the research area of semantic consistency maintenance is rather new, it lacks evaluation tools and theories for its issues. This paper attempts to provide such an evaluation method.

\subsection{Semantic Impact Chain Method}

Our evaluation method tries to build a semantic impact chain (SIC) method to evaluate the impact of a mismatched concept on semantic consistency, such that in a concept supply chain (CSC) if a concept is mismatched with the meaning of its antecedent concept then the semantic inconsistency will happen from then on 
and impact on semantic consistency thereafter. Particularly, a concept supply chain (CSC) can be expressed as follows:

$$
\mathrm{CSC}\left(c_{0}, k, n\right)=\mathrm{M}_{0}\left(c_{0}\right) \rightarrow \mathrm{M}_{1}\left(c_{k}\right) \rightarrow \ldots \rightarrow \mathrm{M}_{n}\left(c_{k}\right)=\mathrm{S}_{n}\left(c_{k}\right),
$$

in which $\mathrm{M}_{0}\left(c_{0}\right)$ is the root concept as a commonly agreed concept collaboratively designed in a community $\mathrm{M}_{0}$ in community level 0 , the $\mathrm{M}_{0}\left(c_{0}\right)$ as a 0 -lelvel mediation concept is localized as $\mathrm{M}_{1}\left(c_{k}\right)$ by $k$ communities in community level 1 . This localization process continues until $\mathrm{M}_{n-1}\left(c_{k}\right)$ as $n-1$ level mediation concepts are localized by $\mathrm{M}_{n}\left(c_{k}\right)$ in $n$ level communities, which are bottom level communities. $\mathrm{M}_{n}\left(c_{k}\right)$ are also called source level concepts.

$\mathrm{CSC}\left(c_{0}, k, n\right)$ follows the concept uses in ConexNet [7], where $\mathrm{M}$ are concept mediation nodes and $\mathrm{S}$ are concept source nodes. The maximum concept number of $\operatorname{CSC}\left(c_{0}, k, n\right)$ supplied by $c_{0}$ is:

$$
\operatorname{CSC}\left(c_{0}, k, n\right)=k^{n} \text {, }
$$

where root concept $c_{0}$ supplies with $k^{n}$ concepts in a $k$-branching tree of depth $n$. It is also called as an n-level concept localization tree with $k^{n}$ localized concepts in $k^{n}$ localization communities.

Special cases can be discussed. If the root level only have 1 level localization, then

$$
\operatorname{CSC}\left(c_{0}, k, 1\right)=k \text {. }
$$

If there are 2 localization levels and for each $1^{\text {st }}$-level localized concept $k_{i}$, there is corresponding $k_{i}$ number of $2^{\text {nd }}$-level localized concepts, then the total number of 2-level localization is:

$$
\operatorname{CSC}\left(c_{0}, k, 2\right)=k+\sum_{1}^{k} k_{i}^{i} \text {. }
$$

With this introduction of $\operatorname{CSC}\left(c_{0}, k, n\right)$, we define a semantic impact chain (SIC) as follows:

$$
\operatorname{SIC}(i, j, k, n)^{\mathrm{CSC}}=\mathrm{M}_{0}\left(c_{0}\right) \rightarrow \mathrm{M}_{\mathrm{i}}\left(c_{k}\right)^{\mathrm{j}} \rightarrow \ldots \rightarrow \mathrm{M}_{n}\left(c_{k}\right),
$$

where a concept $\mathrm{M}_{\mathrm{i}}\left(c_{k}\right)^{\mathrm{j}}$ is mismatched in $\operatorname{CSC}\left(c_{0}, k, n\right)$ at $i$-level with the path of $(0 \ldots j)$, which impacts the semantic consistency of its branching community trees. The maximum impacted concept number of $\operatorname{SIC}(i, j, k, n)^{\mathrm{CSC}}$ that could be semantically inconsistent can be calculated as follows:

$$
\operatorname{SIC}(i, j, k, n)^{\mathrm{CSC}}=k^{n-i},
$$

in which if concept mismatch happens at $i$-level of $(0 \ldots j)$, the impacted concept number is $k^{n-i}$. If $i=n$ at $n$-level, the impacted concept is the localized concept of the impact community itself.

\subsection{SIC Values of COPEX System}

COPEX takes a 1-level concept supply chain architecture with the 0 level of EMF and $1^{\text {st }}$ level of FIRM. With this architecture, the maximum numbers of semantic inconsistent concepts between different locations can be shown as follows:

\begin{tabular}{|l|c|c|c|c|c|}
\hline SIC & $\boldsymbol{n}$ & $\boldsymbol{k}$ & $\boldsymbol{i}$ & $\boldsymbol{k}^{\mathbf{n}-\mathbf{i}}$ & Consistency Maintenance \\
\hline EMF & 1 & 1 & 0 & 1 & P2P collaborative design \\
\hline FIRM & 1 & $k$ & 1 & 1 & D2F collaborative design \\
\hline
\end{tabular}

In this Table, EMF is regarded as a single concept community though there may have many EMF involved. The reason why EMF can present the consistent concepts between different EMF is that all the participated EMF take the P2P collaboration mechanism where they collaboratively design the semantically consistent concepts using the same concept identifiers by explicitly agreeing the meanings conveyed in concept identifiers.

For FIRM, localization process may incur semantic consistency between the local concepts of FIRM and the common concepts of EMF when using D2F collaborative design mechanism, if local concept designers mismatch the meaning of local concepts with the meaning of common concepts. This is important and higher attention should be paid by FIRM concept designers because their localized concepts could affect the semantically consistent transformation of heterogeneous business processes from their firms to other firms. Luckily, the semantic inconsistency effect will be minimized to only affect the firm who makes the mistake since the $k$ is 1 , that is, the inconsistent concept localization by firm only affects itself.

In addition, through the Table, we can also see the meaning mismatch of heterogeneous concepts only affects its lower level communities. This is a good feature of COPEX, that is, it can isolate semantic inconsistencies within a local community and no propagation as a global effect.

To have a better understanding of formula $\operatorname{SIC}(i, j, k, n)^{\mathrm{CSC}}$ that computes the impacted concept number that might be semantically inconsistent, we take the PVC poncho example, shown in Figure 5 of USI, to illustrate the computation. In this example, the maximum concept number of a USI SendInquiry document is 30 and the maximum semantically inconsistent concepts are computed as SIC $(1 \text {, USI, } 1,1)^{\mathrm{CSC}}$ on USI SendInquiry $=30 \times \operatorname{SIC}(1$, USI, 1,1$)^{\mathrm{CSC}}=30 \times k^{n-i}=30 \times 1^{1-1}=1^{0} \times 30=30$. This implies that, for each FIRM, the maximum impacted concept number that could be semantically inconsistent is the maximum number that is mistakenly mismatched by that FIRM. This further implies that to control the impacted concept number is to cautiously map the local concepts of FIRM onto the common concepts of EMF.

\section{CONCLUSION}

In this paper, we studied the semantic consistency maintenance issue between heterogeneous contexts, that is, how a firm' business process of one e-marketplace can be transformed to another firm's business process of another e-marketplace in a semantically consistent way. The proposed solution of this paper uses XML Product Map (XPM) of collaborative concept to represent semantically consistent business processes, and adopts common action concept pool and XPM documents to design heterogeneous business processes that are suitable for heterogeneous business process transformation. We motivated the approach with a realworld PVC poncho trade problem and explained it in architecture of collaborative process design and automatic service provision. We reported the implementation specification within a hybrid human-agent framework, where four layers of system modules are specified. The approach is evaluated based on a new semantic impact chain method particularly for evaluating concept consistency in meaning representation between heterogeneous contexts of business processes.

Our approach has advantages comparing with known solutions. (1) We do not rely on single business process standards that cannot cope with the issue of semantic consistency maintenance, but a set of collaboratively designed and mapped cross-domain business processes for enabling heterogeneous business process transformation. (2) The architecture we provide is collaborative, distributed, role-based and service-oriented. It is highly flexible for many semantically different systems to both join and leave without unfavorable consequences. Technically, the flexibility is reflected in the freedom of multiple e-marketplace participation and multiple levels of personalization in basic concepts (including products), composite document concepts and complex process pattern concepts. (3) The categorization of complex concepts into basic, composiste, action and reified concepts help understand what the essential components are for a generic business process. 
(4) We introduce a common action concept pool to collaboratively design common business processes that are apt for localize and use in heterogeneous business contexts. This pool enables heterogneous business processes to be freely and semantically reassembled using different action concepts. (5) We devise electronic document oriented and PRODUCT MAP based busines processes to ease the integration and transformation of heterogeneous business processes. (6) We use XPM to represent business processes. It helps the separation of business process structure representation from business process concept representation, which enhances the design reusability of both process structures and process concepts. (6) The evaluation method of semantic impact chain we provide helps find possible problems of the designed system in semantic consistency between heterogeneous business processes.

The main limitations of our work are: (1) a considerable large set of system participants should be included for evaluating the impact of possible semantic consistency. (2) XPM specification for business process has not been evolved to include public reusable action logic (i.e. public executables defined in $\langle$ logic $\rangle$ ). Action logics must be designed individually in different firms by themselves when they localized the common business processes.

Based on COPEX approach, there could be many future works that could be extended. For example, in legal aspect, how to guarantee an e-document (e.g. an offer/quotation) is legally binding by following which laws when it is corss two legal territory. In information security aspect, how to guarantee the confidentiality and integrity of the transmitting XPM e-documents In the aspect of international process standard interoperability, how to enable XPM-based COPEX business processes to be interoperable with the exisiting business process standards (currently we simply regard an international standard as a business process specification of a local FIRM).

Nevertheless, in the near future, we only intend to extend the work in the following directions: (1) Provide a demonstratible heterogeneous business process transformer. (2) Provide a demonstratible collaborative business process editor and a demonstratible business process localizer. (3) Publish a stable XPM specification for guiding the design of business process. Yet we hope this work could attract more people to work towards COPEX related research issues.

\section{ACKNOWLEDGMENTS}

The work reported in this paper has been partially supported by University of Macau Research Grand. We also thank anonymous reviewers for their insightful comments.

\section{REFERENCES}

[1] Barthes, R. Elements of Semiology. Hill and Wang, English version 1968.
[2] Borghoff, U. and R. Pareschi (eds). Information Technology for Knowledge Management, Springer, 1998.

[3] Bußler, C. Specifying Enterprise Processes with Workflow Modeling Languages. Concurrent Engineering 4(3) (1996) 261-278.

[4] Davenport, T. and J. Short. Operations Management: The New Industrial Engineering: Information Technology and Business Process Redesign. In Michael Lewis, Nigel Slack (eds) Critical Perspectives on Business and Management,

[5] Goh, C., Madnick, S. and M. Siegel, Context Interchange: Overcoming the Challenges of Large-Scale Interoperable Database Systems in a Dynamic Environment, in: Proc. CIKM'94 (ACM, 1994) 337-346.

[6] Guan, X. and J. Guo. Context-based Translation of Constant Concept Values in E-Business. In: Proc. of $3^{\text {rd }}$ IEEE/IFIP Int'l Conf. in Central Asia on Internet (ICI 2007) (Tashkent, Uzbekistan, September 26 - 28, 2007).

[7] Guo, J. Collaborative Concept Exchange, VDM Publishing: Germany, 2008.

[8] Guo, J. Inter-Enterprise Business Document Exchange. In: Proc. ICEC'06, (ACM Press, 2006) 427-437.

[9] Guo, J. A Transparent Collaborative Integration Approach for Ad Hoc Product Data. In: Proc. CEC'06/EEE'06 (IEEE Computer Society Press 2006).

[10] Guo, J. A Term in Search of the Infrastructure of Electronic Markets. In: Research and Practical Issues of Enterprise Information Systems II Volume 2, IFIP Volume 255, eds. L. Xu, Tjoa A., Chaudhry S. (Boston: Springer 2008) 831-840.

[11] Hallen, L., Johanson, J. and N. Seyed-Mohamed. Interfirm Adaptation in Business Relationships. Journal of Marketing 55(2) (1991) 29-37.

[12] Hepp, M., Leukel, J. and V. Schmitz. A Quantitative Analysis of eCl@ss, UNSPSC, eOTD, and RNTD: Content, Coverage, and Maintenance. In: Proc. ICEBE 2005 (IEEE Computer Society Press, 2005) 572-581.

[13] Innis, R. (ed). Semiotics: An Introductory Anthology. Hutchinson, 1985.

[14] Jung, J., Hur, W., Kang, S. and H. Kim. Business process choreography for B2B collaboration. IEEE Internet Computing 8(1) (2004) 37-45.

[15] Lambert, D. and M. Cooper. Issues in Supply Chain Management. Industrial Marketing Management 29(1) (2000) 6583.

[16] Saussure, F. Course in General Linguistics, McGraw-Hill Book Company, 1966.

[17] Sun, C., Jia, X., Zhang, Y., Yang, Y. and D. Chen, Achieving Convergence, Causality Preservation, and Intention Preservation in Real-Time Cooperative Editing Systems, ACM Transactions on Computer-Human Interaction, 5(1), (1998) 63-108. 